NOTICE: This is the author's version of a work that was accepted for publication in the Journal of Biomechanics. Changes resulting from the publishing process, such as peer review, editing, corrections, structural formatting, and other quality control mechanisms, may not be reflected in this document. Changes may have been made to this work since it was submitted for publication. A definitive version was subsequently published in Journal of Biomechanics Volume 43, Issue 9, June 2010, Pages 1844-184, doi:10.1016/j.jbiomech.2010.02.018.

\title{
Compensation of large motion sensor displacements during long recordings of limb movements
}

\author{
Dominik Karch ${ }^{1}$, Keun-Sun Kim ${ }^{2}$, Katarzyna Wochner ${ }^{2}$, Heike Philippi ${ }^{3}$, \\ Joachim Pietz ${ }^{2}$, Hartmut Dickhaus ${ }^{1}$ \\ ${ }^{1}$ Institute of Medical Biometry and Informatics, University of Heidelberg, Im Neuenheimer Feld 305, 69120 Heidelberg, Germany \\ ${ }^{2}$ Center for Child and Adolescent Medicine, University Hospital Heidelberg \\ ${ }^{3}$ Center of Developmental Medicine, Frankfurt
}

\begin{abstract}
In motion capture applications using electromagnetic tracking systems the process of anatomical calibration associates the technical frames of sensors attached to the skin with the human anatomy. Joint centers and axes are determined relative to these frames. A change of orientation of the sensor relative to the skin renders this calibration faulty. This sensitivity regarding sensor displacement can turn out to be a serious problem with movement recordings of several minutes duration. We propose the "dislocation distance" as a novel method to quantify sensor displacement and to detect gradual and sudden changes of sensor orientation. Furthermore a method to define a so called fixed technical frame is proposed as a robust reference frame which can adapt to a new sensor orientation on the skin. The proposed methods are applied to quantify the effects of sensor displacement of 120 upper and lower limb movement recordings of newborns revealing the need for a method to compensate for sensor displacement. The reliability of the fixed technical frame is quantified and it is shown that trend and dispersion of the dislocation distance can be significantly reduced. A working example illustrates the consequences of sensor displacement on derived angle time series and how they are avoided using the fixed technical frame.
\end{abstract}

\section{Introduction}

Electromagnetic tracking systems have gained widespread use in biomechanical applications [Meskers et al., 1998; Umberger et al., 1999; Biryukova et al., 2000; Stokdijk et al., 2000; Longworth et al., 2006; Mills et al., 2007; van Andel et al., 2008]. Their small sensors render possible applications like the analysis of infant movements [Karch et al., 2008]. One movement sensor can measure 6 degrees of freedom (DOF) which enables the direct tracking of the orientation of an object. However, this advantage can turn out to be a serious drawback when it comes to long movement recordings (> 3 min): During anatomical calibration [Cappozzo et al., 2005] the technical frame of a sensor (STF) gets associated with joint positions and axes. A so called anatomical frame $(\mathrm{AF})$ is defined relative to the STF so that the orientation of the segment can be determined for every instant when the orientation of the STF is measured. It is crucial that the orientation of the sensor does not change relative to the segment. If that happens the calibration is no longer valid which leads to kinematic crosstalk or unusable data.
During long recordings gradual or sudden changes of sensor orientation might occur caused by external factors like the contact of the infant's hand or foot with a sensor. Hitherto there have been no quantitative criteria to quantify this sensor displacement (which is not to be confused with the issue of soft tissue artifacts [Cerveri et al., 2005; Leardini et al., 2005; Riemer et al., 2008]). Thus a method is needed to quantify and compensate for errors. The methodological contribution of this article is twofold:

- A method is presented for the quantification of sensor displacement.

- A fixed technical frame is introduced which can be used as a robust reference frame for an arbitrarily defined anatomical frame.

We apply these ideas to upper and lower limb recordings of 120 infants. The results are illustrated by an example which shows how kinematic crosstalk in hand joint angle time series can be compensated for. 


\section{Methods}

\subsection{Quantitative indicator for sensor displace- ment}

In order to quantify sensor displacement directly we need a reference frame associated with the same segment. Since such a frame is not available we take advantage of a second STF which is attached to an adjacent segment. If a point ${ }^{g} p$ in the global reference system $g$ exists which is common to both technical frames with orientation matrices $R_{\mathrm{s}_{1}}$ and $R_{\mathrm{S}_{2}}$ and position vectors $p_{\mathrm{s}_{1}}$ and $p_{\mathrm{s}_{2}}$ its constant relative position in the technical frames can be determined as ${ }^{\mathrm{s}_{1}} p$ and ${ }^{\mathrm{s}_{2}} p$, respectively. The global distance between these relative points is

$$
d(t)=\left\|\left(p_{\mathrm{s}_{1}}(t)+R_{\mathrm{s}_{1}}(t)^{\mathrm{s}_{1}} p\right)-\left(p_{\mathrm{s}_{2}}(t)+R_{\mathrm{s}_{2}}(t)^{\mathrm{s}_{2}} p\right)\right\|
$$

It should be constantly zero in the ideal case. If one of these sensors changes its orientation on the skin, the global position ${ }^{\mathrm{g}} p_{1}$ translates. Consequently the distance $d(t)$ increases. Hence an increasing distance can be used as an indicator for sensor displacement. We will refer to $d(t)$ as dislocation distance in the remainder.

The center of rotation (COR) between two segments [Biryukova et al., 2000] can be used as a constant reference point which approximately complies with the requirement of a common fixed point.

\subsection{Compensation of changes of sensor orienta- tion}

Typically the AF is directly associated with the technical frame of the sensor:

$$
\mathrm{STF} \rightarrow \mathrm{AF}
$$

If a change of sensor orientation occurs the $\mathrm{AF}$ is no longer aligned correctly. Therefore we introduce an intermediate frame whose pose is constant with respect to the tracked segment. This so called fixed technical frame (FTF) can then be used as a reference for the anatomical frame:

$$
\mathrm{STF} \rightarrow \mathrm{FTF} \rightarrow \mathrm{AF}
$$

In order to define such a frame which adapts to a changed sensor orientation three non-aligned points are needed, which fulfill one of the following properties:

a) The point stays constant with respect to the segment and the STF as well. The position of the sensor on the skin is used as a first point.

b) The point stays constant with respect to the segment and can be calculated from movement data. This applies to the cors of the two adjacent joints of the segment.

The positions of a COR in the adjacent STFs $\left({ }^{\mathrm{S}_{1}} p_{d}\right.$ as the distal point in the STF of the first sensor and ${ }^{\mathrm{S}_{2}} p_{p}$ as the proximal point in the STF of the second sensor) are determined so that their global distance is minimal. If these points are calculated as points with constant position in the respective STFs as in [Biryukova et al., 2000; Gamage and Lasenby, 2002] they suffer from the problems of dislocation. Therefore we define them as variable reference points ${ }^{\mathrm{s}_{1}} p_{d}(t)$ and ${ }^{\mathrm{s}_{2}} p_{p}(t)$. Their instantaneous positions at $t_{0}$ are calculated using the movement data from a temporal window $w$

$$
\underset{\mathrm{s}_{1} p_{d}\left(t_{0}\right),{ }^{2} p_{p}\left(t_{0}\right)}{\operatorname{argmin}} \frac{1}{w} \int_{w}\left({ }^{\mathrm{g}} p_{d}(t)-{ }^{\mathrm{g}} p_{p}(t)\right)^{2} \mathrm{~d} t
$$

The position vectors ${ }^{\mathrm{s}} p_{p}(t)$ and ${ }^{\mathrm{s}} p_{d}(t)$ in the sTF can be used to define the FTF (see Fig. 1). When this frame is determined at calibration time $t_{0}$, the orientation $R_{A}$ of the AF can be associated with it

$$
R_{F \rightarrow A}=R_{F}^{T}\left(t_{0}\right) R_{A}\left(t_{0}\right)
$$

$R_{F \rightarrow A}$ maps the FTF to the AF. Hence the pose of the AF at any time can be calculated from the FTF

$$
R_{A}(t)=R_{F}(t) R_{F \rightarrow A}
$$

\subsection{Experimental setup}

Limb motions of 120 infants at an age between one and three months were recorded with an electromagnetic tracking device (3D Guidance medsAFE ${ }^{\mathrm{TM}}$, Ascension Technology, sample frequency $50 \mathrm{~Hz}$, working volume $16 \times 40 \times 40 \mathrm{~cm}^{3}$ ). Four sensors each were attached to the arm and the leg as described in [Karch et al., 2008] (see Fig. 2). The experimental protocol was explained to all parents and their written informed consent was obtained.

The evaluation is done in two steps:

a) The precision of the estimation of the variable reference points needed for the compensation method is evaluated by quantifying the movements of each point in its respective STF during recordings without displacement. If there is no displacement they should maintain their position. This movement is 

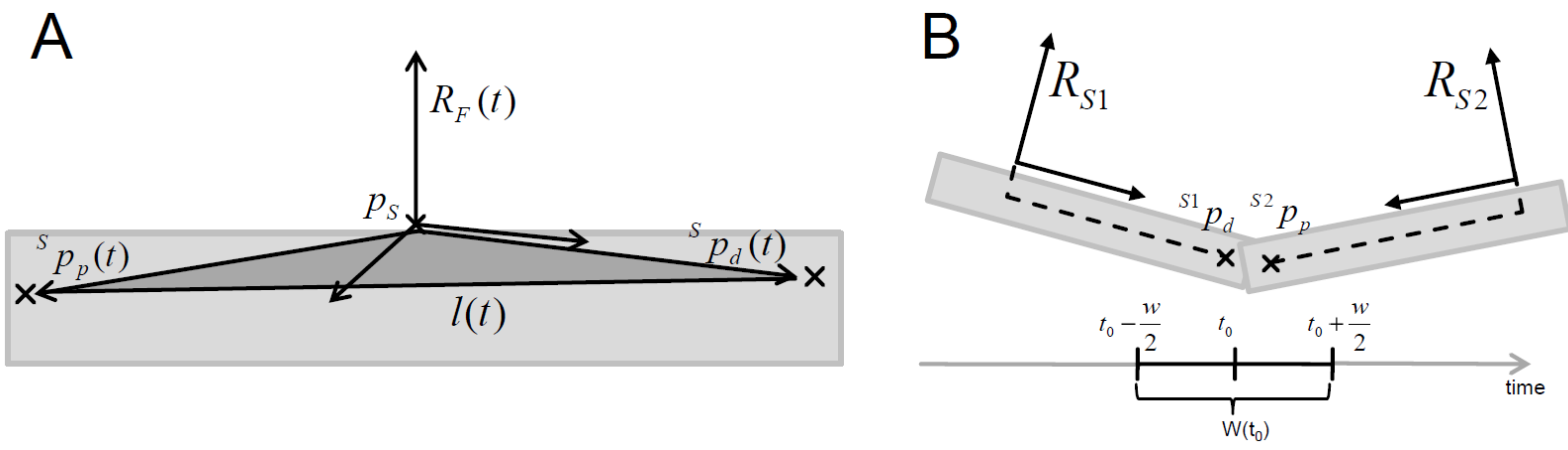

Figure 1: (A) Definition of a fixed technical frame based on the position vectors of the proximal and distal reference points ${ }^{\mathrm{s}} p_{p}(t)$ and ${ }^{\mathrm{s}} p_{d}(t)$ in the technical frame $R_{F}(t)$. The distance $1(\mathrm{t})$ has to be approximately constant. (B) Determination of reference points. ${ }^{\mathrm{s} 1} p_{d}\left(t_{0}\right)$ and ${ }^{\mathrm{s} 2} p_{p}\left(t_{0}\right)$ are determined as variable points in the respective technical frames $R_{\mathrm{s} 1}$ and $R_{\mathrm{s} 2}$ so that their distance during the time span $W\left(t_{0}\right)$ is minimal. $W(t)$ is symmetrical around $t$.
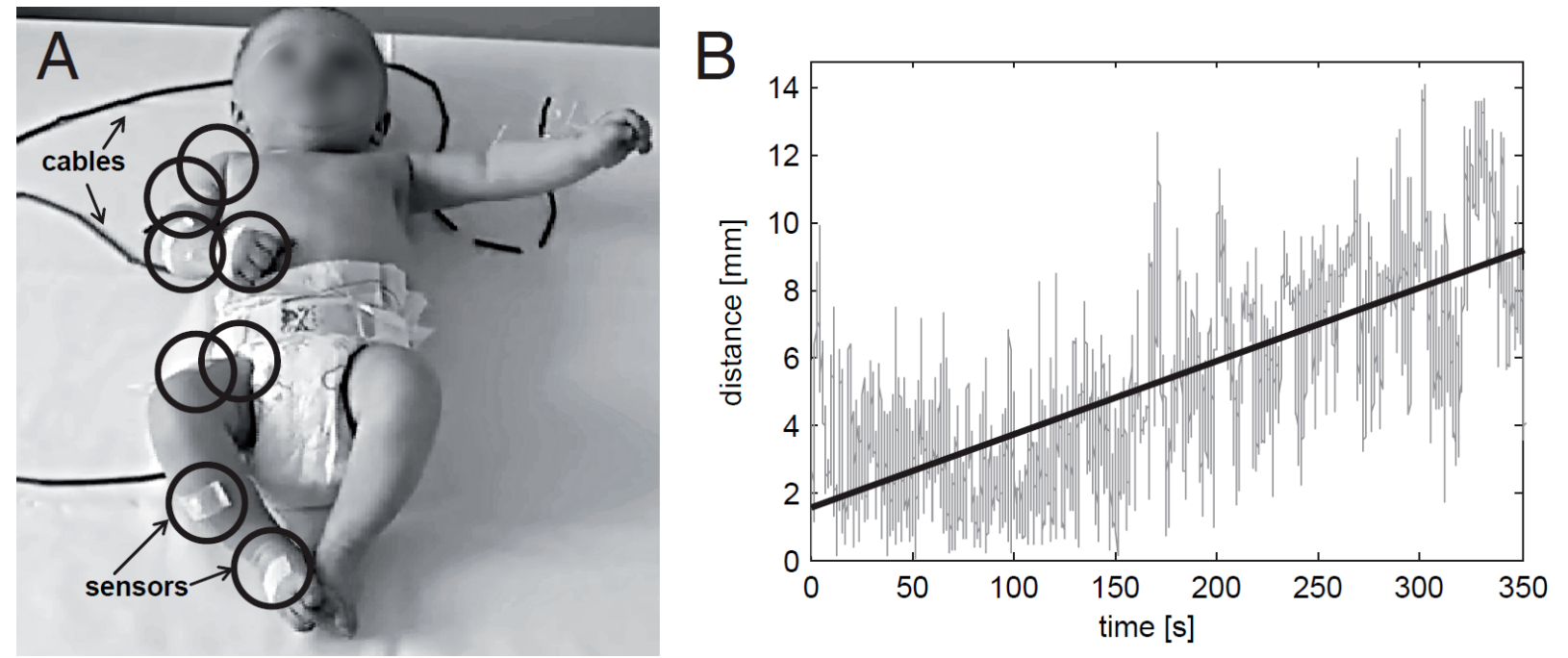

Figure 2: (A) Infant during motion recording. The circles depict the positions where sensors are attached to the skin with eudermic patches. (B) Dislocation distance at the knee exhibiting an increasing trend (black regression line) due to a gradual change of sensor orientation. 
described by a distribution $\Delta p$ with

$$
\Delta p(t)={ }^{\mathrm{s}} p(t)-{ }^{\mathrm{s}} \bar{p}
$$

where ${ }^{\mathrm{S}} p(t)$ is the position of the reference point in its STF at time ${ }^{\mathrm{S}} \bar{p}$ and is its average position. For each $\Delta p$ the mean value $\Delta p_{\text {mean }}$ and the max value $\Delta p_{\max }$ are calculated quantifying the mean and max deviation of the reference point, respectively.

b) The amount of dislocation using constant (see section 2.1) and variable (see section 2.2) reference points is quantified by the absolute trend of $d(t)$ and the mean absolute deviation from this trend (MAD).

\section{Results}

To analyze the precision of the reference points we examined the dispersion of their positions in the respective STFs. We included only recordings with small dislocation distances (trend $<3 \mathrm{~mm} / 5 \mathrm{~min}$ and $\mathrm{MAD}<4 \mathrm{~mm}$ ). For all reference points Table 1 shows the mean of $\Delta p_{\text {mean }}$ and $\Delta p_{\max }$ of all recordings. The last row shows the resulting deviation $\alpha$ for the FTFs for typical segment lengths which can be calculated with

$$
\alpha=\tan ^{-1} \frac{\Delta p_{1}+\Delta p_{2}}{l}
$$

where $\Delta p_{1}$ and $\Delta p_{2}$ are the deviation of the first and second reference point and $l$ is the length of the segment. The mean deviation of $\alpha=4^{\circ}$ is a low value considering that the sTF can easily rotate by $90^{\circ}$. In order to quantify the amount of sensor displacement Table 2 shows the median values of trend and MAD using constant and variable reference points, respectively. Fig. 3 shows box plots of the distribution of the trends. For the constant reference points the trends at all joints show distributions with a small median but with large dispersion and lots of outliers. In contrast the plots of the other group exhibit medians near zero with very small dispersion. A sign test showed that the differences between the methods are significant (each $\mathrm{p}$-value at least $<0.02$ ).

Fig. 2 shows an example of gradual displacement. The trend line reveals that the mean of $d(t)$ is increasing. Fig. 4 shows an example for a sudden change: $d(t)$ of wrist and elbow increase almost simultaneously which is a clear indication that the forearm sensor has changed its orientation at one moment.

Fig. 5 illustrates the consequences of this sudden displacement for the derived angle time series and its compensation. In the upper part a sudden jump at $t_{1}$ shifts the mean by more than $45^{\circ}$. Contrarily the lower part

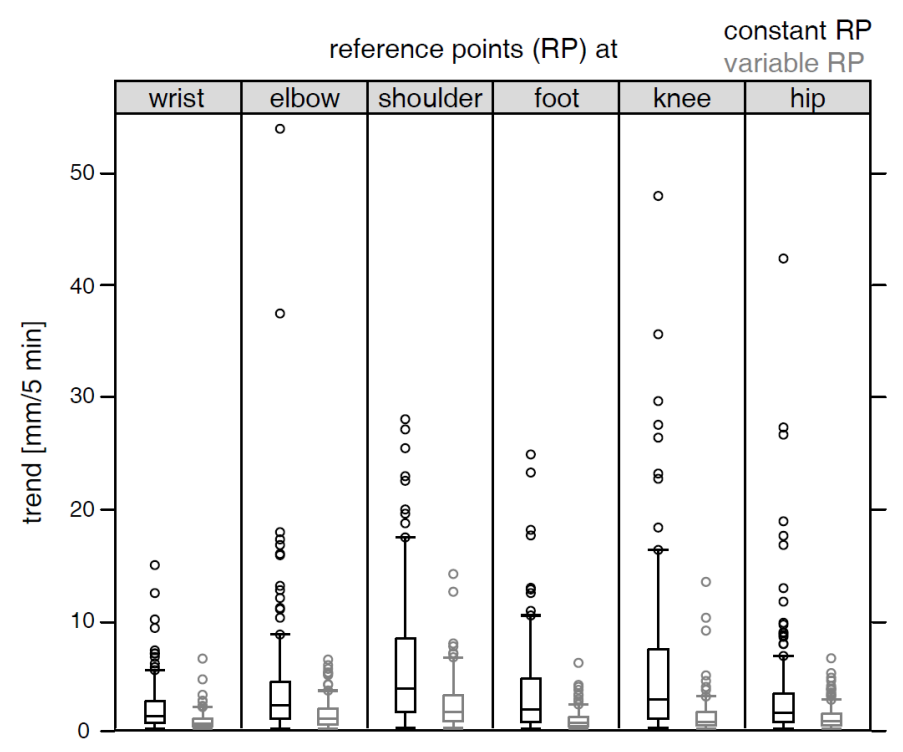

Figure 3: Box plots of the absolute trend of dislocation distance $d(t)$ at different joints using constant (black) and variable (gray) reference points.

demonstrates how the use of the FTF prevents these effects.

\section{Discussion}

We introduced the dislocation distance (see Eq. 1) which can be used to detect changes of sensor orientation. In the case of a sudden change the synchronously rising slope allows to exactly identify the time and the involved sensor. The proposed FTF can be used as a robust reference frame for arbitrarily defined (see e.g. [Wu et al., 2002; Ehrig et al., 2007]) AFs. Fig. 5 illustrates its advantages: as long as there is no change of sensor orientation (before $t_{1}$ ) it makes no difference whether one associates the AF directly with the STF or indirectly with the FTF. The benefit of adopting the latter becomes evident after $t_{1}$ : If there is a direct association the AF is misaligned after the displacement. If the indirect association is used this reference frame can adapt to the new sensor orientation. There are three limitations: First of all, the FTF can only be defined for a limb segment if sensors are attached to the two adjacent segments. Secondly, this approach can only be applied sensibly if a recording lasts several minutes. Thirdly, the time spans between sudden changes of sensor orientation have to be sufficiently long so that the reference points in Eq. 2 can be calculated reliably. E.g. if there is a sudden change after the first $30 \mathrm{~s} R_{F \rightarrow A}$ in Eq. 5 will be inaccurate. In this case the anatomical calibration should be repeated. This described problem does not exist with optical tracking systems. Using stereopho- 
A: constant reference points
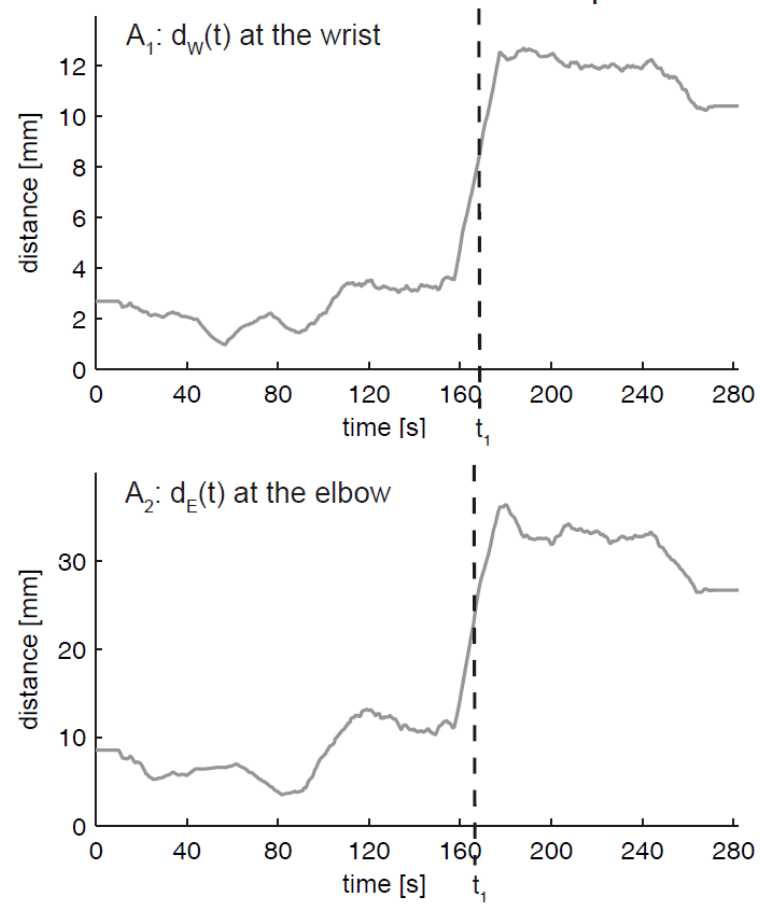

B: variable reference points
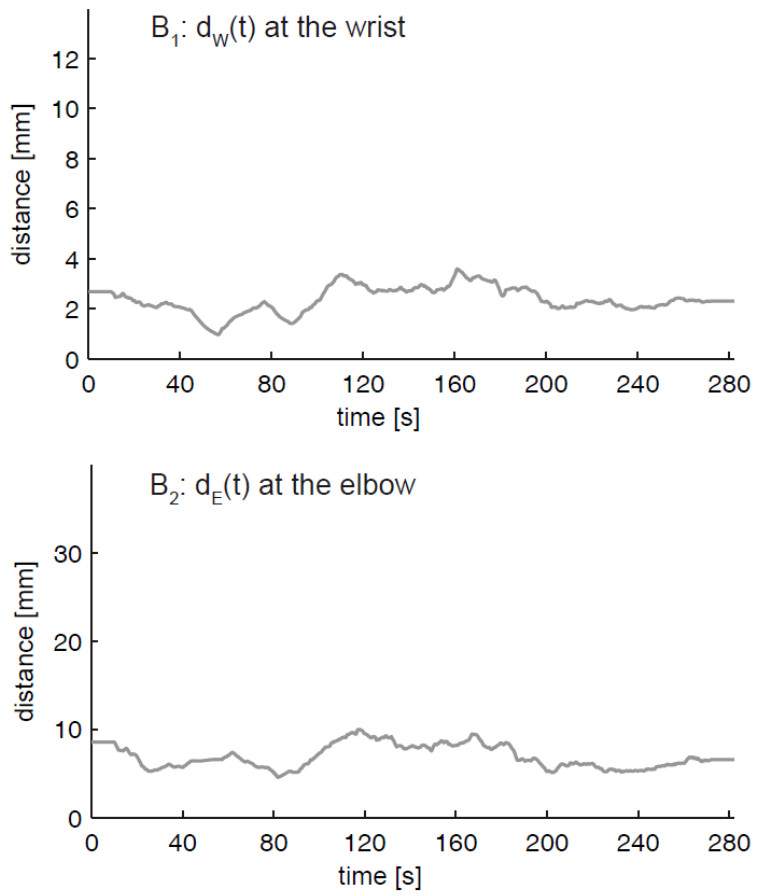

Figure 4: Detection of a sudden change of orientation of the forearm sensor using dislocation distances $d_{w}(t)$ at the wrist and $d_{e}(t)$ at the elbow (both smoothed with a moving average filter of $60 \mathrm{~s}$ ). (A) A sudden slope appears almost synchronously. The estimated time $t_{1}$ of this event is marked with dashed lines. (B) Variable reference points adapt their positions in the respective technical frames. Hence $d_{w}(t)$ and $d_{e}(t)$ remain low.

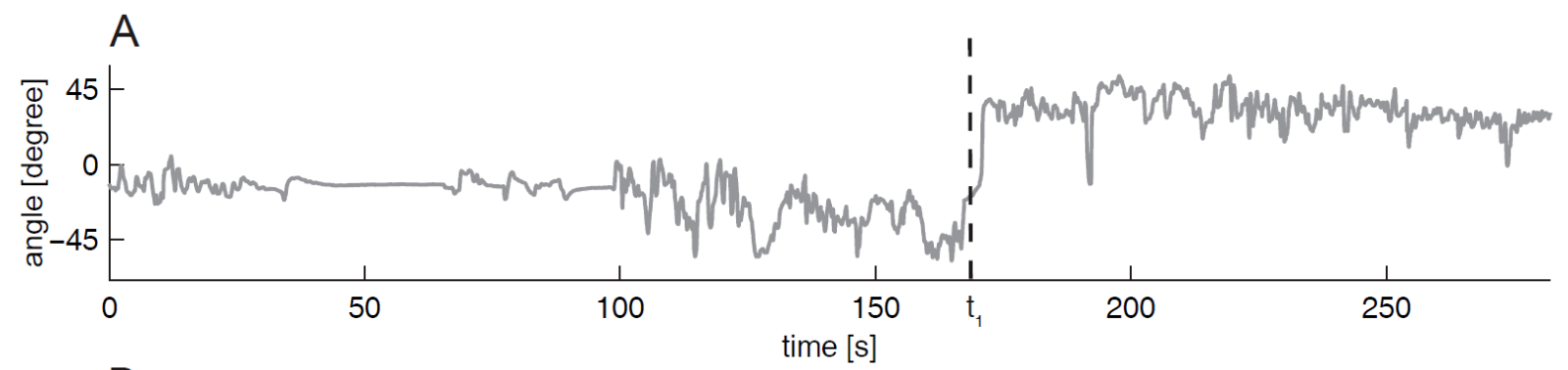

B

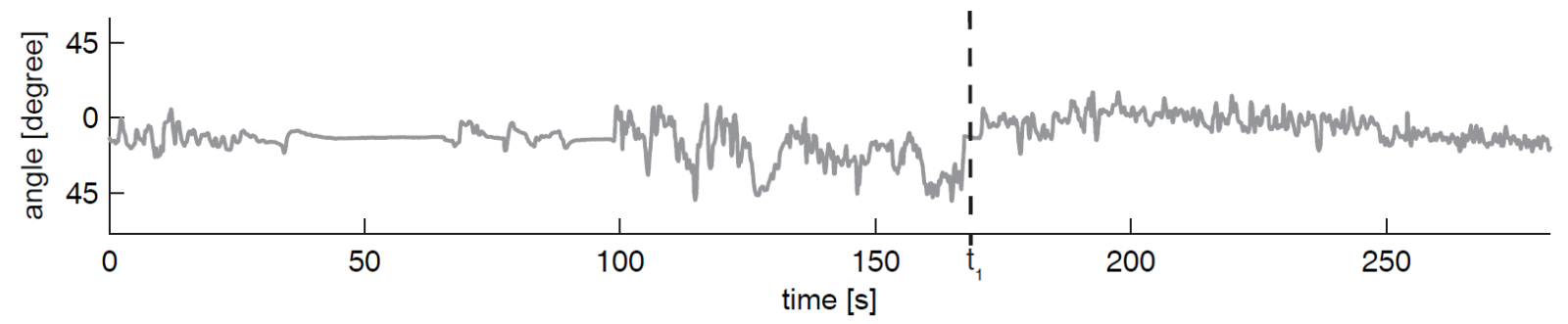

Figure 5: Effects of a sudden change of orientation of the forearm sensor which has been detected at $t_{1}$ (see Fig. 4) on the derived abduction/adduction angle time series of the hand. (A) Anatomical frame relative to sensor technical frame. The misalignment of the anatomical frame after $t_{1}$ leads to kinematic crosstalk. (B) Anatomical frame relative to fixed technical frame. The fixed technical frame adapts to the new sensor orientation. 
Table 1: Precision of the calculation of reference points and resulting deviation of the corresponding fixed technical frames (FTF). $\mathrm{n}$ is the number of included recordings without displacement for the respective point.

\begin{tabular}{|c|c|c|c|c|c|c|c|c|}
\hline & \multirow{2}{*}{$\begin{array}{c}\text { Shoulder } \\
(\mathrm{n}=41) \\
\text { dist }\end{array}$} & \multicolumn{2}{|c|}{$\begin{array}{l}\text { Elbow } \\
(\mathrm{n}=70)\end{array}$} & \multirow{2}{*}{$\begin{array}{c}\text { Wrist } \\
(\mathrm{n}=100) \\
\text { prox }\end{array}$} & \multirow{2}{*}{$\begin{array}{c}\text { Hip } \\
(\mathrm{n}=86) \\
\text { dist }\end{array}$} & \multicolumn{2}{|c|}{$\begin{array}{c}\text { Knee } \\
(\mathrm{n}=60)\end{array}$} & \multirow{2}{*}{$\begin{array}{c}\text { Foot } \\
(\mathrm{n}=77) \\
\text { prox }\end{array}$} \\
\hline & & prox & dist & & & prox & dist & \\
\hline mean of $\Delta p_{\text {MEAN }}[\mathrm{mm}]$ & 3.1 & 2.9 & 2.9 & 2.6 & 2.5 & 3.1 & 3.0 & 2.8 \\
\hline mean of $\Delta p_{\operatorname{MAX}}[\mathrm{mm}]$ & 5.5 & 6.7 & 6.7 & 5.1 & 4.6 & 6.3 & 6.3 & 5.6 \\
\hline FTF (mean segment length) & \multicolumn{2}{|c|}{ upper $\operatorname{arm}(78 \mathrm{~mm})$} & \multicolumn{2}{|c|}{ forearm $(71 \mathrm{~mm})$} & \multicolumn{2}{|c|}{ thigh $(84 \mathrm{~mm})$} & \multicolumn{2}{|c|}{ lower leg $(101 \mathrm{~mm})$} \\
\hline mean $(\max )$ deviation $\alpha\left[^{\circ}\right]$ & \multicolumn{2}{|c|}{$4(9)$} & \multicolumn{2}{|c|}{$4(9)$} & \multicolumn{2}{|c|}{$4(7)$} & \multicolumn{2}{|c|}{$3(7)$} \\
\hline
\end{tabular}

Table 2: Median of absolute trend and dispersion of the dislocation distance $d(t)$ using constant and variable reference points for 120 infants.

\begin{tabular}{lllll}
\hline & \multicolumn{2}{c}{ Constant reference points } & \multicolumn{2}{c}{ Variable reference points } \\
& $\begin{array}{l}\text { Trend: Median } \\
\text { (mean abs. dev.) }[\mathrm{mm}]\end{array}$ & $\begin{array}{l}\text { Mean absolute deviation } \\
\text { from the trend: median [mm] }\end{array}$ & $\begin{array}{l}\text { Trend: Median } \\
\text { (mean abs. dev.) }[\mathrm{mm}]\end{array}$ & $\begin{array}{l}\text { Mean absolute deviation } \\
\text { from the trend: median [mm] }\end{array}$ \\
\hline Shoulder & $3.6(4.0)$ & 4.0 & $1.5(1.5)$ & 3.3 \\
Elbow & $2.1(3.2)$ & 2.4 & $0.9(0.9)$ & 1.9 \\
Wrist & $1.1(1.4)$ & 1.4 & $0.9(0.9)$ & 1.1 \\
Hip & $1.4(2.7)$ & 1.8 & $0.7(0.8)$ & 1.7 \\
Knee & $2.6(4.4)$ & 1.9 & $0.6(0.9)$ & 1.5 \\
Foot & $1.7(2.7)$ & 1.6 & $0.5(0.6)$ & 1.2 \\
\hline
\end{tabular}

togrammetry only position data can be measured, i.e. the orientation of a segment has to be reconstructed [Cappozzo et al., 1997]. This methodology is more robust. In a way our approach of defining a FTF mimics the way technical frames are defined in this field.

\section{Conflict of interest}

This project has been funded by the Dietmar-HoppStiftung, Walldorf, Germany. The Dietmar-HoppStiftung did not have any influence on this work.

\section{References}

Biryukova, E. V., Roby-Brami, A., Frolov, A. A., and Mokhtari, M. (2000). Kinematics of human arm reconstructed from spatial tracking system recordings. Journal of Biomechanics, 33:985-995.

Cappozzo, A., Cappello, A., Della Croce, U., and Pensalfini, F. (1997). Surface-Marker Cluster Design Criteria for 3-D Bone Movement Reconstruction. IEEE Transactions on Biomedical Engineering, 44(12):1165-1174.

Cappozzo, A., Della Croce, U., Leardini, A., and
Chiari, L. (2005). Human movement analysis using stereophotogrammetry: Part 1: theoretical background. Gait E Posture, 21(2):186-196.

Cerveri, P., Pedotti, A., and Ferrigno, G. (2005). Kinematical models to reduce the effect of skin artifacts on marker-based human motion estimation. Journal of Biomechanics, 38(11):2228-2236.

Ehrig, R. M., Taylor, W. R., Duda, G. N., and Heller, M. O. (2007). A survey of formal methods for determining functional joint axes. Journal of Biomechanics, 40:2150-2157.

Gamage, S. S. H. U. and Lasenby, J. (2002). New least squares solutions for estimating the average centre of rotation and the axis of rotation. Journal of Biomechanics, 35(1):87-93.

Karch, D., Kim, K. S., Wochner, K., Pietz, J., Dickhaus, H., and Philippi, H. (2008). Quantification of the segmental kinematics of spontaneous infant movements. Journal of Biomechanics, 41(13):2860-2867.

Leardini, A., Chiari, L., Della Croce, U., and Cappozzo, A. (2005). Human movement analysis using stereophotogrammetry Part 3. Soft tissue arti- 
fact assessment and compensation. Gait \& Posture, 21:212-225.

Longworth, R., Chockalingam, N., and Redmond, A. C. (2006). An enhanced protocol to reduce error in electromagnetic tracking of first metatarsophalangeal joint motions. Gait \& Posture, 23(3):391-394.

Meskers, C. G. M., Vermeulen, H. M., de Groot, J. H., van Der Helm, F. C. T., and Rozing, P. M. (1998). 3D shoulder position measurements using a six-degreeof-freedom electromagnetic tracking device. Clinical Biomechanics, 13(4-5):280-292.

Mills, P. M., Morrison, S., Lloyd, D. G., and Barrett, R. S. (2007). Repeatability of 3D gait kinematics obtained from an electromagnetic tracking system during treadmill locomotion. Journal of Biomechanics, 40(7):1504-1511.

Riemer, R., Hsiao-Wecksler, E. T., and Zhang, X. (2008). Uncertainties in inverse dynamics solutions: A comprehensive analysis and an application to gait. Gait E Posture, 27(4):578-588.

Stokdijk, M., Nagels, J., and Rozing, P. M. (2000). The glenohumeral joint rotation centre in vivo. Journal of Biomechanics, 33:1629-1636.

Umberger, B. R., Nawoczenski, D. A., and Baumhauer, J. F. (1999). Reliability and validity of first metatarsophalangeal joint orientation measured with an electromagnetic tracking device. Clinical Biomechanics, 14(1):74-76.

van Andel, C. J., Wolterbeek, N., Doorenbosch, C. A. M., Veeger, D., and Harlaar, J. (2008). Complete 3D kinematics of upper extremity functional tasks. Gait \& Posture, 27(1):120-127.

Wu, G., Siegler, S., Allard, P., Kirtley, C., Leardini, A., Rosenbaum, D., Whittle, M., D'Lima, D., Cristofolini, L., Witte, H., Schmidt, O., and Stokes, I. (2002). ISB recommendation on definitions of joint coordinate systems of various joints for the reporting of human joint motion - Part I: ankle, hip, and spine. Journal of Biomechanics, 35:543-548. 